\title{
Liapounoff's vector measure theorem in Banach spaces and applications to general equilibrium theory
}

\author{
Michael Greinecker • Konrad Podczeck
}

Received: 10 September 2013 / Accepted: 16 September 2013 / Published online: 26 September 2013 (C) SAET 2013

\begin{abstract}
We present a result on convexity and weak compactness of the range of a vector measure with values in a Banach space, based on the Maharam classification of measure spaces. Our result extends a recent result of Khan and Sagara (Illinois J. Math. 2013). We apply our result to integration of Banach space valued correspondences and to the core-Walras equivalence problem in coalitional exchange economies with an infinite-dimensional commodity space.
\end{abstract}

Keywords Liapounoff's theorem · Vector measures · Correspondences · Blocking power of small coalitions · Core-Walras equivalence $\cdot$ Coalitional economies

JEL Classification $\quad \mathrm{C} 02 \cdot \mathrm{C} 60 \cdot \mathrm{C} 71 \cdot \mathrm{D} 51$

\section{Introduction}

In various contexts, it was observed that measure spaces of uncountable Maharam type allow for useful constructions that are not available for the unit interval with Lebesgue measure; see, e.g., Scott (1967), Hoover and Keisler (1984), Rustichini and Yannelis

We are grateful to Nicholas Yannelis for helpful suggestions and comments.

M. Greinecker $(\bowtie)$

Institut für Wirtschaftstheorie, Universität Innsbruck, SOWI-Gebäude, Universitätsstraße 15, 6020 Innsbruck, Austria

e-mail: michael.greinecker@uibk.ac.at

K. Podczeck

Institut für Volkswirtschaftslehre, Universität Wien,

Oskar-Morgenstern-Platz 1, 1090 Wien, Austria

e-mail: konrad.podczeck@univie.ac.at 
(1991), Podczeck (2008), or Keisler (2009). Continuing this line, Khan and Sagara (2013) recently established a version of Liapounoff's theorem for vector measures with values in a Banach space.

The main result in Khan and Sagara (2013) says that if $(T, \Sigma, \mu)$ is a totally finite measure space and $G$ is a $\mu$-continuous countably additive vector measure defined on $\Sigma$ with values in a Banach space $X$, then the range of $G$ is a convex and weakly compact set in $X$ if $\mu$ is Maharam-type-homogeneous with Maharam type strictly larger than the algebraic dimension of $X$.

In this note, we sharpen this result of Khan and Sagara (2013). In particular, we remove the hypothesis of Maharam-type-homogeneity, and, in the condition on the codomain of a vector measure, replace the algebraic dimension of a Banach space by the cardinal of some point-separating family of continuous linear functionals. The latter has drastic consequences; see the comments after Corollary 5 below.

We provide two proofs of our main result. Our first proof is very short. It reveals that versions of Liapounoff's theorem based on the Maharam classification of measure spaces are, in fact, a straightforward consequence of Knowles' version of Liapounoff's theorem in the weak topology (Knowles 1975), which we use in the form as stated in Diestel and Uhl (1977, Theorem IX.1.4).

Our second proof is essentially measure-theoretic and works without the extreme point arguments on which the proof of Diestel and Uhl (1977, Theorem IX.1.4) is based. Instead, it makes use of an idea introduced by Maharam (1942) in the proof of her classification result for measure algebras.

We illustrate the usefulness of our main result with three applications. First, we establish results on the convexity of the Aumann integral of a correspondence taking values in a Banach space, following the lines of the classical proof by Richter (1963) for the case of a finite-dimensional codomain. Second, we show that the result in Schmeidler (1972) on blocking power of small coalitions can be extended to infinite-dimensional commodity spaces, covering in particular models of commodity differentiation. Finally, we establish a result on core-Walras equivalence in coalitional exchange economies in the spirit of Vind (1964) when the commodity space is an order-continuous Banach lattice.

\section{Notation and terminology}

If $A$ is a set, \# $(A)$ denotes its cardinal. As usual, $\omega$ denotes the least infinite cardinal, and $\omega_{1}$ the least uncountable cardinal.

If $X$ is a Banach space, then $X^{*}$ denotes its dual space, and for a set $U \subseteq X$, dens $(U)$ denotes the density of $U$, i.e., the least cardinal of any set $V \subseteq U$ which is dense in $U$. Thus if $X \neq\{0\}, X$ is separable if and only if $\operatorname{dens}(X)=\omega$.

Let $(T, \Sigma, \mu)$ be a totally finite measure space. If $E \subseteq T, \mu_{E}$ denotes the subspace measure on $E$ defined from $\mu$. By $\mathfrak{A}_{\mu}$ we denote the measure algebra of $\mu$, and for $a \in \mathfrak{A}_{\mu}$, by $\mathfrak{A}_{a}$ the principal ideal in $\mathfrak{A}_{\mu}$ generated by $a$. If $\kappa$ is an infinite cardinal, we say that $(T, \Sigma, \mu)$, or the measure $\mu$, is $\kappa$-atomless if for each non-zero $a \in \mathfrak{A}_{\mu}, \mathfrak{A}_{a}$ 
has Maharam type at least $\kappa .{ }^{1}$ Note that " $\omega$-atomless" means just "atomless" in the usual sense. Note also that being " $\kappa$-atomless" does not imply being Maharam-typehomogeneous.

We shall make use of the following facts.

Fact 1 Let $(T, \Sigma, \mu)$ be a totally finite measure space, and $\kappa$ an infinite cardinal. Then $\mu$ is $\kappa$-atomless if and only if $\operatorname{dens}\left(L_{1}\left(\mu_{E}\right)\right) \geq \kappa$ for each $E \in \Sigma$ with $\mu(E)>0$.

(To see this, apply Fremlin $(2004,331 Y($ e) or $365 Y)$ to the non-zero principal ideals of the measure algebra of $(T, \Sigma, \mu)$.)

Fact 2 If $X$ is an infinite-dimensional Banach space, then $\operatorname{dens}(X)$ is equal to the least cardinal of a set $A \subseteq X$ such that $A$ separates the points of $X^{*}$.

(If $A \subseteq X$ is dense, then $A$ separates the points of $X^{*}$. Conversely, let $A \subseteq X$ separate the points of $X^{*}$. Then $\overline{\operatorname{span}} A=X$ by the Hahn-Banach theorem; in particular, $\#(A)$ must be infinite if $X$ is infinite-dimensional. Now, writing $F$ for the set of (finite) linear combinations with rational coefficients of the members of $A, F$ is dense in $\overline{\operatorname{span}} A$, and $\#(F)=\#(A)$ if $\#(A)$ is infinite.)

Finally, we settle some terminology concerning vector measures. Let $(T, \Sigma, \mu)$ be a totally finite measure space, and $X$ a Banach space. A function $G: \Sigma \rightarrow X$ is called a vector measure if it is additive, i.e., if $G(E \cup F)=G(E)+G(F)$ for all $E$, $F \in \Sigma$ with $E \cap F=\emptyset$. A vector measure $G: \Sigma \rightarrow X$ is called countably additive if $G\left(\bigcup_{n=0}^{\infty} E_{n}\right)=\sum_{n=0}^{\infty} G\left(E_{n}\right)$ in the norm of $X$ whenever $\left\langle E_{n}\right\rangle$ is a disjoint sequence in $\Sigma$, and is called $\mu$-continuous if $\lim _{\mu(E) \rightarrow 0} G(E)=0$. (Of course, a $\mu$-continuous vector measure $G: \Sigma \rightarrow X$ is countably additive.) If $f \in L_{\infty}(\mu)$ and $G: \Sigma \rightarrow X$ is a $\mu$-continuous countably additive vector measure, then $\int f \mathrm{~d} G$ denotes the Bartle integral. Given a $\mu$-continuous countably additive $G: \Sigma \rightarrow X$, the Bartle integral is a continuous linear operator from $L_{\infty}(\mu)$ to $X$, which on simple functions is defined as $\int f \mathrm{~d} G=\sum_{i=1}^{n} \alpha_{i} G\left(E_{i}\right)$ if $f=\sum_{i=1}^{n} \alpha_{i} 1_{E_{i}}$, and via continuous linear extension is defined on all of $L_{\infty}(\mu)$; see Diestel and Uhl (1977, pp. 5 and pp. 56), and note for this reference that a countably additive vector measure has a bounded range Diestel and Uhl (1977 Corollary I.1.19).

\section{The vector measure theorem}

Here is our version of Liapounoff's theorem.

Theorem Let $(T, \Sigma, \mu)$ be a totally finite measure space, $X$ a Banach space, and $G: \Sigma \rightarrow X$ a $\mu$-continuous countably additive vector measure. Let $\kappa$ be an infinite cardinal and assume that $\mu$ is $\kappa$-atomless and that there is a family $\left\langle x_{i}^{*}\right\rangle_{i \in I}$ in $X^{*}$, with $\#(I)<\kappa$, which separates the points of $\overline{\operatorname{span}} G(\Sigma)$. Then for every $E \in \Sigma$, $\{G(A \cap E): A \in \Sigma\}$ is a weakly compact and convex set in $X$.

\footnotetext{
1 Recall that the Maharam type of a Boolean algebra $\mathfrak{A}$ is the least cardinal of any set $\mathcal{H} \subseteq \mathfrak{A}$ such that the order-closed subalgebra of $\mathfrak{A}$ generated by $\mathcal{H}$ is $\mathfrak{A}$ itself, and that any principal ideal of a Boolean algebra can be viewed as a Boolean algebra in its own right. For a comprehensive treatment of measure algebras, see Fremlin (2004). For the needs of the present paper, the material in Section 2.2 of Podczeck (2008) suffices.
} 
Proof By Diestel and Uhl (1977 Theorem IX.1.4), we need to show that for any $E \in \Sigma$ with $\mu(E)>0$, the operator $T_{E}: L_{\infty}\left(\mu_{E}\right) \rightarrow X$ given by $T_{E}(f)=\int_{E} f \mathrm{~d} G_{E}$ is not an injection, where $G_{E}$ is the restriction of $G$ to $\{A \cap E: A \in \Sigma\} .^{2}$ Fix any such $E$. By Diestel and Uhl (1977 Lemma IX.1.3), $T_{E}$ is weak*-weakly continuous, so for each $i \in I, x_{i}^{*} T_{E}$ is a weak*-continuous linear functional on $L_{\infty}\left(\mu_{E}\right)$ and may be identified with an element of $L_{1}\left(\mu_{E}\right)$. Note that $T_{E}$ takes its values in $\overline{\operatorname{span}} G(\Sigma)$ (use the definition of the Bartle integral described above).

The hypothesis on $\mu$ implies that $L_{1}\left(\mu_{E}\right)$ is infinite-dimensional. Moreover, by Fact 1 , dens $\left(L_{1}\left(\mu_{E}\right)\right) \geq \kappa$. Thus, by Fact 2 , the family $\left\langle x_{i}^{*} T_{E}\right\rangle_{i \in I}$ in $L_{1}\left(\mu_{E}\right)$ cannot separate the points of $L_{\infty}\left(\mu_{E}\right)$, as \#(I) $<\kappa$, so $T_{E}$ is not an injection, as $T_{E}\left(L_{\infty}\left(\mu_{E}\right)\right) \subseteq \overline{\operatorname{span}} G(\Sigma)$ and $\left\langle x_{i}^{*}\right\rangle_{i \in I}$ separates the points of $\overline{\operatorname{span}} G(\Sigma)$.

The theorem yields several corollaries, where the interesting case is $X$ being infinitedimensional. For the first corollary, just recall that the density of any Banach space $X$ is at least as large as the least cardinal of any $A \subseteq X^{*}$ such that $A$ separates the points of $X$ (cf. Fabian et al. 2001, page 358).

Corollary 1 Let $(T, \Sigma, \mu)$ be a totally finite measure space, and $X$ a Banach space. Assume that for some uncountable cardinal $\kappa, \mu$ is $\kappa$-atomless with $\kappa>\operatorname{dens}(X)$. Then for every $\mu$-continuous countably additive vector measure $G: \Sigma \rightarrow X$ and every $E \in \Sigma,\{G(A \cap E): A \in \Sigma\}$ is a weakly compact and convex set in $X$.

The next corollary of our theorem is more general; see Remark 1 below.

Corollary 2 Let $(T, \Sigma, \mu)$ be a totally finite measure space, $X$ a Banach space, and $G: \Sigma \rightarrow X$ a $\mu$-continuous countably additive vector measure. Assume that for some uncountable cardinal $\kappa, \mu$ is $\kappa$-atomless with $\kappa>\operatorname{dens}(G(\Sigma)$. Then for every $E \in \Sigma,\{G(A \cap E): A \in \Sigma\}$ is a weakly compact and convex set in $X$.

Proof The linear combinations with rational coefficients of the members of a dense subset of $G(\Sigma)$ are dense in $\overline{\text { span }} G(\Sigma)$, and therefore the hypothesis implies that $\kappa>\operatorname{dens}(\overline{\operatorname{span}} G(\Sigma))$. Apply Corollary 1 with $X=\overline{\operatorname{span}}$.

The following special case of Corollary 1 is the content of Theorem 4.1 in Khan and Sagara (2013).

Corollary 3 Let $(T, \Sigma, \mu)$ be a totally finite measure space, and $X$ a separable Banach space. If $\mu$ is $\omega_{1}$-atomless, then for any $\mu$-continuous countably additive vector measure $G: \Sigma \rightarrow X,\{G(A \cap E): A \in \Sigma\}$ is a weakly compact and convex set in $X$ for every $E \in \Sigma$.

If $X$ is a dual Banach space, say $X=Y^{*}$, then any dense subset of $Y$ separates the points of $X$. Thus the above theorem also implies the following result.

Corollary 4 Let $(T, \Sigma, \mu)$ be a totally finite measure space, and $X$ a dual Banach space, say $X=Y^{*}$. Assume that for some uncountable cardinal $\kappa, \mu$ is $\kappa$-atomless

\footnotetext{
${ }^{2}$ Note for this reference that $L_{\infty}\left(\mu_{E}\right)$ can be identified with the subspace of $L_{\infty}(\mu)$ consisting of the elements vanishing off $E$.
} 
with $\kappa>\operatorname{dens}(Y)$. Then for any $\mu$-continuous countably additive vector measure $G: \Sigma \rightarrow X,\{G(A \cap E): A \in \Sigma\}$ is a weakly compact and convex set in $X$ for every $E \in \Sigma$.

A particular case of Corollary 4 is noted next.

Corollary 5 Let $(T, \Sigma, \mu)$ be a totally finite measure space, and $X$ the dual of a separable Banach space. If $\mu$ is $\omega_{1}$-atomless, then for any $\mu$-continuous countably additive vector measure $G: \Sigma \rightarrow X,\{G(A \cap E): A \in \Sigma\}$ is a weakly compact and convex set in $X$ for every $E \in \Sigma$.

Remark 1 The condition in Corollary 2 is more general than that in Corollary 1 . In fact, the range of a Banach space valued countably additive vector measure defined on a $\sigma$-algebra is always relatively weakly compact (Diestel and Uhl 1977 Corollary I.2.7), and there are plenty of non-separable Banach spaces in which every weakly compact subset is (norm) separable. E.g., weakly compact subsets of $C(K)$ - the space of continuous real-valued functions on a compact Hausdorff space $K$, endowed with its sup-norm - are (norm) separable whenever $K$ carries a Radon measure with full support (Rosenthal 1969 Theorem 1.4).

Remark 2 Corollary 1 improves Theorem 5.1 in Khan and Sagara (2013) where the measure space domain $(T, \Sigma, \mu)$ is required to be Maharam-type-homogeneous, and the Maharam-type of $\mu$ to be strictly larger than the algebraic dimension of the codomain of a vector measure. Note that any infinite cardinal is possible as the density of a Banach space; e.g., dens $\left(\ell_{2}(\kappa)\right)=\kappa$ if $\kappa$ is any infinite cardinal. On the other hand, the algebraic dimension of an infinite-dimensional Banach space is at least as large as the cardinality of the continuum $\mathfrak{c}=2^{\omega}$ (Mackey 1945 Theorem I-1), and by Easton's (1970) theorem, the only restriction the usual axioms of set theory put on the cardinal of $2^{\omega}$ is that it has uncountable cofinality and does not exceed the cardinal of $2^{\omega_{1}}$.

Remark 3 Another improvement of our results over those in Khan and Sagara (2013) is provided by Corollary 5. E.g., let $X=M[0,1]$, the space of bounded signed Borel measures on $[0,1]$ with the total variation norm. Then $X$ is non-separable, but $X=Y^{*}$ where $Y=C[0,1]$, the space of continuous functions on $[0,1]$ with the sup-norm, which is a separable. For the conclusion of Corollary 5, the results in Khan and Sagara (2013) would require $\kappa$ to be strictly larger than the algebraic dimension of $M[0,1]$, i.e., $\kappa>2^{\omega}$, while, as shown by our Corollary $5, \kappa=\omega_{1}$ suffices.

Remark 4 For any infinite cardinal $\kappa$, the " $<$ " in our theorem cannot be replaced by " $\leq$ ". This may be seen as follows (cf. the famous example in Uhl 1969). Fix any infinite cardinal $\kappa$. Let $\mu$ be the usual measure on $\{0,1\}^{\kappa}$, and $\Sigma$ its domain. Define $G: \Sigma \rightarrow L_{1}(\mu)$ by setting $G(E)=1_{E}$ for each $E \in \Sigma$. Then $G$ is a $\mu$ continuous countably additive vector measure such that $G(\Sigma)$ is not a convex subset of $L_{1}(\mu)$. In addition, $\overline{\operatorname{span}} G(\Sigma)=L_{1}(\mu)$. Now by the choice of $\mu, \mu$ is Maharamtype-homogeneous with Maharam type $\kappa$; thus, in the terminology of this note, $\mu$ is $\kappa$-atomless. Also, dens $\left(L_{1}(\mu)\right)=\kappa$, and of course, $L_{1}(\mu)$ is infinite-dimensional. By Fabian et al. (2001 Example (v), page 358), the Banach space $L_{1}(\mu)$ is weakly 
compactly generated, so by Fabian et al. (2001 Theorem 11.3), the weak*-density of its dual is also $\kappa$. As $L_{1}(\mu)$ is infinite-dimensional, the least cardinal of any pointseparating family of continuous linear functionals on $L_{1}(\mu)$ is $\kappa$, too. ${ }^{3}$

Remark 5 As shown in Lemma 4 in Podczeck (2008), ${ }^{4}$ if $X$ is any infinite-dimensional Banach space and $(T, \Sigma, \mu)$ is any totally finite measure space such that $\mu$ is not $\omega_{1^{-}}$ atomless, then there is a Bochner-integrable function $f: T \rightarrow X$ such that the set $\left\{\int_{E} f \mathrm{~d} \mu: E \in \Sigma\right\}$ is not a convex subset of $X{ }^{5}$ Translating this into vector measure terms yields directly the following fact (see also Khan and Sagara 2013, Lemma 4.1 and Theorem 4.2 (ii) $\Rightarrow$ (i)).

Proposition 1 Let $(T, \Sigma, \mu)$ be a totally finite measure space, and $X$ an infinitedimensional Banach space. If $\mu$ is not $\omega_{1}$-atomless, then there is $\mu$-continuous countably additive vector measure $G: \Sigma \rightarrow X$ such that the set $\{G(A): A \in \Sigma\}$ is not a convex subset of $X$.

Combining this with Corollary 3 we obtain the following statement.

Proposition 2 Let $(T, \Sigma, \mu)$ be a totally finite measure space, and X a separable infinite-dimensional Banach space. In order that for any $\mu$-continuous countably additive vector measure $G: \Sigma \rightarrow X$, the set $\{G(A \cap E): A \in \Sigma\}$ is a weakly compact and convex subset of $X$ for every $E \in \Sigma$, it is both necessary and sufficient that $\mu$ is $\omega_{1}$-atomless.

Remark 6 Let $(T, \Sigma, \mu)$ be a totally finite measure space, and $X$ any Banach space. If $\mu$ is atomless, then by Kluvánek (1973 Theorem 1) the weak closure of the range of every $\mu$-continuous countably additive vector measure $G: \Sigma \rightarrow X$ is convex. Consequently, Proposition 1 implies that if $X$ is infinite-dimensional and $\mu$ is atomless but not $\omega_{1}$-atomless, then there exists a $\mu$-continuous countably additive vector measure $G: \Sigma \rightarrow X$ with a range that is not weakly compact.

Remark 7 By what was pointed out in Remark 1, an analog of the necessity part of Proposition 2 formulated by replacing, with an uncountable cardinal $\kappa$, "separable infinite-dimensional" in the condition on $X$ by $\operatorname{dens}(X)=\kappa$, and $\omega_{1}$-atomless by $\kappa^{+}$-atomless, where $\kappa^{+}$is the cardinal successor of $\kappa$, is wrong.

\section{An alternative proof of the theorem for $\kappa$ uncountable}

If the range of a vector measure is not finite-dimensional, then our theorem requires the cardinal $\kappa$ to be uncountable. For such a $\kappa$, we can present an alternative proof

\footnotetext{
${ }^{3}$ The weak ${ }^{*}$-density of the dual $X^{*}$ of any infinite-dimensional Banach space $X$ is equal to the least cardinal of any subset of $X^{*}$ separating the points of $X$, which follows similarly as Fact 2 above.

4 The construction in the proof of that lemma follows that in the proof of Diestel and Uhl (1977 Corollary IX.1.6).

${ }^{5}$ Recall the standard fact that if $(T, \Sigma, \mu)$ is a totally finite measure space, $X$ a Banach space, and $f: T \rightarrow$ $X$ is Bochner integrable, then the indefinite Bochner integral of $f$ is a $\mu$-continuous countably additive vector measure.
} 
of our theorem. It is important to note that this alternative proof does not apply to the classical situation of a finite-dimensional vector measure which is just assumed to be atomless; see Remark 9 below. On the other hand, the proof we will present in this section does not rely on Knowles' version of Liapounoff's theorem in the weak topology (Diestel and Uhl 1977 Theorem IX.1.4), and in particular, is not based on noninjectivity of certain linear operators. Altogether, this reveals that, in the terminology of the statement of our theorem, $\kappa>\#(I)$ with $\kappa$ uncountable but $\#(I)$ allowed to be infinite is not simply an analog of $\omega>\#(I)$ - the situation of the classical Liapounoff theorem-for larger cardinals.

In this section, we need some additional notation and terminology. Let $(T, \Sigma, \mu)$ be a totally finite measure space, with measure algebra $\mathfrak{A}_{\mu}$. For any $E \in \Sigma$, we write $E^{\circ}$ for the element of $\mathfrak{A}_{\mu}$ determined by $E$. For the following, note that for each $E \in \Sigma$ with $\mu(E)>0$, the principal ideal $\mathfrak{A}_{E^{\circ}}$ in $\mathfrak{A}_{\mu}$ generated by $E^{\circ}$ may be viewed as a Boolean algebra in its own right and may be written as $\mathfrak{A}_{E^{\circ}}=\left\{(E \cap F)^{\circ}: F \in \Sigma\right\}$. Note also that if a sub- $\sigma$-algebra $\Sigma_{1}$ of $\Sigma$ is generated by a set $\mathcal{A} \subseteq \Sigma_{1}$, then the order-closed subalgebra of $\mathfrak{A}_{\mu}$ generated by the $\mu$-equivalence classes of the members of $\mathcal{A}$ is just the set $\left\{F^{\circ}: F \in \Sigma_{1}\right\}$ (use Fremlin $2004321 \mathrm{X}(\mathrm{b})$ ). Finally, $\Sigma$ is called relatively atomless over a sub- $\sigma$-algebra $\Sigma_{1}$ if for each $E \in \Sigma$ with $\mu(E)>0, \mathfrak{A}_{E^{\circ}} \neq$ $\left\{(E \cap H)^{\circ}: H \in \Sigma_{1}\right\}$. (This definition just puts the definition of "relatively atomless" as stated in Fremlin (2004 331A) for abstract Boolean algebras into terms of measure spaces.)

For convenience of reference, we recall two measure theoretic facts. The first one may be deduced from Fremlin (2004 313M(b)).

Fact 3 Let $(T, \Sigma, \mu)$ be a totally finite measure space, and $\Sigma_{1}$ a sub- $\sigma$-algebra of $\Sigma$ generated by a family $\mathcal{A} \subseteq \Sigma_{1}$. Then for each $E \in \Sigma$ with $\mu(E)>0$, the order-closed subalgebra of $\mathfrak{A}_{E^{\circ}}$ generated by $\left\{(E \cap A)^{\circ}: A \in \mathcal{A}\right\}$ includes $\left\{(E \cap H)^{\circ}: H \in \Sigma_{1}\right\}$.

For the next fact, see Fremlin (2004 331B). This fact provided the basis on which our alternative method of proof relies.

Fact 4 Let $(T, \Sigma, \mu)$ be a totally finite measure space, $\Sigma_{1}$ a sub- $\sigma$-algebra of $\Sigma$, and $v: \Sigma_{1} \rightarrow \mathbb{R}$ an additive functional such that $0 \leq v(H) \leq \mu(H)$ for every $H \in \Sigma_{1}$. Suppose that $\Sigma$ is relatively atomless over $\Sigma_{1}$. Then there is an $F \in \Sigma$ such that $\nu(H)=\mu(H \cap F)$ for every $H \in \Sigma_{1}$.

Lemma 1 Let $\kappa$ be an infinite cardinal, $(T, \Sigma, \mu)$ a $\kappa$-atomless totally finite measure space, and $\Sigma_{1}$ a sub- $\sigma$-algebra of $\Sigma$ generated by a family $\mathcal{A} \subseteq \Sigma_{1}$ with $\#(\mathcal{A})<\kappa$. Then given any $\Sigma$-measurable $f: T \rightarrow[0,1]$ there is an $F \in \Sigma$ such that $\int_{H} f \mathrm{~d} \mu=$ $\mu(H \cap F)$ for each $H \in \Sigma_{1}$.

Proof Define $v: \Sigma_{1} \rightarrow \mathbb{R}_{+}$by $v(H)=\int_{H} f \mathrm{~d} \mu$ for $H \in \Sigma_{1}$. Evidently $v$ is additive with $0 \leq v(H) \leq \mu(H)$ for each $H \in \Sigma_{1}$. In view of Fact 3, the hypotheses imply that $\Sigma$ is relatively atomless over $\Sigma_{1}$. The claim now follows from Fact 4.

Lemma 2 Let $\kappa$ be an infinite cardinal, $(T, \Sigma, \mu)$ a $\kappa$-atomless totally finite measure space, $f: T \rightarrow[0,1]$ a $\Sigma$-measurable function, and $\Sigma_{1}$ a sub- $\sigma$-algebra of $\Sigma$ generated by a family $\mathcal{A} \subseteq \Sigma_{1}$ with $\#(\mathcal{A})<\kappa$. Then there is an $F \in \Sigma$ such that $\int_{T} h f \mathrm{~d} \mu=\int_{F} h \mathrm{~d} \mu$ for each integrable and $\Sigma_{1}$-measurable $h: T \rightarrow \mathbb{R}$. 
Proof Let $F$ be chosen according to Lemma 1, so that $\int_{T} h f \mathrm{~d} \mu=\int_{F} h \mathrm{~d} \mu$ holds whenever $h$ is the characteristic function of some $H \in \Sigma_{1}$. It follows that this equality also holds for $h$ being any $\Sigma_{1}$-measurable simple function, and thus for $h$ being any $\mu$-integrable and $\Sigma_{1}$-measurable function.

Alternative proof of the theorem for $\kappa$ uncountable. We need to consider only the case $E=T$. (Let $E \in \Sigma$. If $\mu(E)=0, G$ vanishes on $E$. If $\mu(E)>0$, then $\mu_{E}$ is $\kappa$-atomless, for the same $\kappa$ for which $\mu$ is, directly by the definition of this property; further, $A \subseteq B \subseteq X$ implies dens $(A) \leq \operatorname{dens}(B)$.) Now by Diestel and Uhl (1977 Lemma IX.1.3),

$$
\overline{\operatorname{co}} G(\Sigma)=\left\{\int_{T} f \mathrm{~d} G: 0 \leq f \leq 1, f \in L_{\infty}(\mu)\right\},
$$

and by Diestel and Uhl (1977 Corollary I.2.7), $G(\Sigma)$ is relatively weakly compact, so $\overline{\mathrm{co}} G(\Sigma)$ is weakly compact by the Krel̆n-Smulian theorem. Thus it suffices to show that given any $f \in L_{\infty}(\mu)$ with $0 \leq f \leq 1$ there is an $F \in \Sigma$ with $G(F)=\int_{T} f \mathrm{~d} G$. Pick any such $f$.

Invoking the family $\left\langle x_{i}^{*}\right\rangle_{i \in I}$ hypothesized, note that for each $i \in I, x_{i}^{*} G$ is a realvalued $\mu$-continuous signed measure, and therefore has a Radon-Nikodym derivative $h_{i} \in L_{1}(\mu)$ which we identify with one of its versions. Now each $h_{i}$ is measurable for some countably generated sub- $\sigma$-algebra of $\Sigma$. Noting that $\#(I) \cdot \omega<\kappa$, because $\kappa$ is assumed to be uncountable, we can therefore find a sub- $\sigma$-algebra $\Sigma_{1}$ of $\Sigma$ such that each $h_{i}$ is $\Sigma_{1}$-measurable and such that $\Sigma_{1}$ is generated by a family $\mathcal{A} \subseteq \Sigma_{1}$ with $\#(\mathcal{A})<\kappa$. By Lemma 2, we can find an $F \in \Sigma$ such that $\int_{T} h_{i} f \mathrm{~d} \mu=\int_{F} h_{i} \mathrm{~d} \mu$ for each $i \in I$. Now for each $i \in I$ we have

$$
x_{i}^{*} \int_{T} f \mathrm{~d} G=\int_{T} f \mathrm{~d} x_{i}^{*} G=\int_{T} h_{i} f \mathrm{~d} \mu=\int_{F} h_{i} \mathrm{~d} \mu=x_{i}^{*} G(F),
$$

showing that $G(F)=\int_{T} f \mathrm{~d} G$, as $\left\langle x_{i}^{*}\right\rangle_{i \in I}$ separates the points of $\overline{\mathrm{co}} G(\Sigma)$.

Remark 8 The heart of the proof as given in this section is Lemma 1. It depends on Fact 4, which originated as part of the proof in Maharam (1942). Exploiting the fact stated in this lemma is what allows to bypass the usual extreme point arguments in proofs of Liapounoff's theorem, which go back to Lindenstrauss (1996).

Remark 9 We said above that the method of the proof given in this section does not cover the classical situation where the Banach space $X$ is finite-dimensional and $(T, \Sigma, \mu)$ is just assumed to be atomless, i.e., $\omega$-atomless in the terminology of this note. To see this, note that for $\kappa=\omega$ the inequality $\#(I) \cdot \omega<\kappa$ is of course wrong

\footnotetext{
${ }^{6}$ For this equality to be valid, the full hypothesis in Diestel and Uhl (1977 Lemma IX.1.3) that $G(E \cap F)=0$ if and only if $\mu(E)=0$ is not needed; the part requiring $\mu$-continuity of $G$ suffices, as may be seen form the proofs of Diestel and Uhl (1977 Lemma IX.1.3 and Corollary I.2.7).
} 
unless $\#(I)=0$. But this strict inequality is necessary in order to apply Lemma 1 and, a fortiori, Lemma 2.

In order to apply the alternative proof to the classical case, one would therefore need to replace the strict inequality in Lemma 1 by a weak inequality. But this is impossible. Let $T=[0,1], \Sigma=\Sigma_{1}$ the Borel $\sigma$-algebra of $[0,1], \mu$ be Lebesgue measure restricted to $\Sigma$, and $f$ be the constant function taking value $1 / 2$. If the conclusion of Lemma 1 would hold in this case, there would be an $F \in \Sigma$ such that $\int_{H} f \mathrm{~d} \mu=\mu(H) / 2=\mu(H \cap F)$ for all $H \in \Sigma$. But then we would have $\mu(F)=1 / 2$, and on the other hand, setting $H=F, \mu(F)=\mu(F) / 2$, which is absurd.

\section{Applications}

Some applications of the above results require an intermediate step of the following kind, which could be of independent interest.

Lemma 3 Let $(T, \Sigma, \mu)$ be a totally finite measure space, $X$ a Banach space, and $G, F: \Sigma \rightarrow X$ two $\mu$-continuous countably additive vector measures. Let $E \in \Sigma$ and $0<\alpha<1$. Then there is an $H \subseteq \Sigma$ with $H \subseteq E$ such that $\alpha G(E)+(1-\alpha) F(E)=$ $G(H)+F(E \backslash H)$ if any of the following conditions hold.

(a) $\mu$ is $\omega_{1}$-atomless and there are separable closed linear subspaces $S_{1}$ and $S_{2}$ of $X$ such that $G(\Sigma) \subseteq S_{1}$ and $F(\Sigma) \subseteq S_{2}$.

(b) For some uncountable cardinal $\kappa, \mu$ is $\kappa$-atomless with $\kappa>\operatorname{dens}(X)$.

(c) $X$ is a dual space, say $X=Y^{*}$, and for some uncountable cardinal $\kappa, \mu$ is $\kappa$-atomless with $\kappa>\operatorname{dens}(Y)$.

Proof The parallel product $(G, F): \Sigma \rightarrow X \times X$, i.e., $(G, F)(A)=(G(A), F(A))$ for every $A \in \Sigma$, is again a $\mu$-continuous countably additive vector measure. If (a) holds then $(F, G)$ can be viewed as a vector measure taking values in the separable Banach space $S_{1} \times S_{2}$. For (b), note that dens $(X \times X)=\operatorname{dens}(X)$, and for (c) note that dens $(Y \times Y)=\operatorname{dens}(Y)$ and that $(Y \times Y)^{*}$ may be identified with $X \times X$. Thus, under any of the three conditions, by Corollaries 3, 1, and 4, respectively, there is an $H \in \Sigma$ with $H \subseteq E$ such that $\alpha(G(E), F(E))=(G(H), F(H))$. Now $F(E \backslash H)=$ $F(E)-F(H)=(1-\alpha) F(E)$, so $G(H)+F(E \backslash H)=\alpha G(E)+(1-\alpha) F(E)$.

\subsection{Integrals of correspondences}

Let $(T, \Sigma, \mu)$ be a totally finite measure space, $X$ a Banach space, and $\varphi: T \rightarrow 2^{X}$ a correspondence. The set of Bochner integrals of all Bochner integrable selections of $\varphi$ is the Aumann-Bochner integral of $\varphi$, the set of Pettis integrals of all Pettis integrable selections of $\varphi$ is the Aumann-Pettis integral of $\varphi$, and if $X$ is a dual Banach space, then the set of Gelfand integrals of all Gelfand integrable selections of $\varphi$ is the Aumann-Gelfand integral of $\varphi$.

The correspondence $\varphi$ is called integrably bounded, if for some integrable function $\rho: T \rightarrow \mathbb{R}_{+}, \sup \{\|x\|: x \in \varphi(t)\} \leq \rho(t)$ for almost all $t \in T$.

To deal with the Gelfand integral of a correspondence, we need some preparation. 
Lemma 4 Let $(T, \Sigma, \mu)$ be a totally finite measure space, $X$ a dual Banach space, and $f: T \rightarrow X$ a Gelfand integrable function. Suppose that for some integrable function $\rho: T \rightarrow \mathbb{R}_{+},\|f(t)\| \leq \rho(t)$ for almost all $t \in T$. Then the indefinite Gelfand integral of $f$ is a $\mu$-continuous (norm-) countably additive vector measure.

Proof Write $G: \Sigma \rightarrow X$ for the indefinite Gelfand integral of $f$. Clearly the function $G$ is additive. Suppose $\left\langle E_{n}\right\rangle_{n \in \mathbb{N}}$ is a sequence in $\Sigma$ with $\mu\left(E_{n}\right) \rightarrow 0$. By hypothesis, we have $X=Y^{*}$ for some Banach space $Y$; write $B_{Y}$ for the closed unit ball in $Y$. Note that for any $y \in B_{Y}$, we have $|y f(t)| \leq\|f(t)\| \leq \rho(t)$ for almost all $t \in T$. Thus, using the definition of the Gelfand integral,

$$
\begin{aligned}
\left\|g\left(E_{n}\right)\right\| & =\sup \left\{\left|y G\left(E_{n}\right)\right|: y \in B_{Y}\right\}=\sup \left\{\left|\int_{E_{n}} y f(t) \mathrm{d} \mu(t)\right|: y \in B_{Y}\right\} \\
& \leq \sup \left\{\int_{E_{n}}|y f(t)| \mathrm{d} \mu(t): y \in B_{Y}\right\} \leq \int_{E_{n}} \rho(t) \mathrm{d} \mu(t) \rightarrow 0
\end{aligned}
$$

as $n \rightarrow \infty$. Thus $G$ is $\mu$-continuous, therefore also countably additive.

Proposition 3 Let $(T, \Sigma, \mu)$ be a totally finite measure space, $X$ a Banach space, and $\varphi: T \rightarrow 2^{X}$ a correspondence.

(a) If $\mu$ is $\omega_{1}$-atomless then the Aumann-Bochner integral of $\varphi$ is convex.

(b) If for some uncountable cardinal $\kappa, \mu$ is $\kappa$-atomless with $\kappa>\operatorname{dens}(X)$, then the Aumann-Pettis integral of $\varphi$ is convex.

(c) Suppose $X$ is a dual space, say $X=Y^{*}$, and that $\varphi$ is integrably bounded. If for some uncountable cardinal $\kappa, \mu$ is $\kappa$-atomless with $\kappa>\operatorname{dens}(Y)$, then the Aumann-Gelfand integral of $\varphi$ is convex.

Proof The indefinite Pettis integral of a Pettis integrable $f: T \rightarrow X$ and, in particular, the indefinite Bochner integral of a Bochner integrable $f: T \rightarrow X$ are $\mu$-continuous countably additive vector measures (Diestel and Uhl 1977 Theorem II.3.5). In the context of (c), Lemma 4 shows that the same is true for the indefinite Gelfand integral of a Gelfand integrable selection $f: T \rightarrow X$ of $\varphi$. Note also that the range of the indefinite Bochner integral of a Bochner integrable function $f: T \rightarrow X$ is included in a separable subspace of $X$.

The proposition now follows from Lemma 3, with $E$ there replaced by $T$, noting that if $g, f$ are any two selections of $\varphi$, then for any $H \subseteq T$, the function $1_{H} g+1_{T \backslash H} f$ is a selection of $\varphi$ as well.

Remark 10 Part (a) of Proposition 3 is not new. See Theorem 1 in Podczeck (2008) and Theorem 6.1 in Khan and Sagara (2013). Theorem 1 in Podczeck (2008) also contains a converse; whenever $(T, \Sigma, \mu)$ is not $\omega_{1}$-atomless and $X$ infinite-dimensional, there is a correspondence $\phi: T \rightarrow 2^{X}$ with non-convex Aumann-Bochner integral.

The special case of Proposition 3(c) where $Y$ is separable is also shown in Theorem 3 in Podczeck (2008). That theorem shows in addition that whenever $(T, \Sigma, \mu)$ is not 
$\omega_{1}$-atomless and $Y$ is separable and infinite-dimensional, there is a correspondence $\phi: T \rightarrow 2^{X}=2^{Y^{*}}$ with non-convex Aumann-Gelfand integral.

Remark 11 See Yannelis (1991) for several results on Banach space valued correspondences and their use in economics. In particular, versions of Fatou's lemma for sequences of such correspondences may be found in this reference.

\subsection{Blocking by small coalitions}

For atomless exchange economies with a finite-dimensional commodity space, Schmeidler (1972) showed that if an allocation can be blocked by some non-negligible coalition, then it can be blocked by non-negligible coalitions of arbitrarily small measure. The argument given by Schmeidler (1972) is based on the fact that, by the classical Liapounoff theorem, an integrable $\mathbb{R}^{n}$-valued function defined on an atomless measure space defines a vector measure with convex range.

If an analog property holds with an infinite-dimensional codomain, then the argument in Schmeidler (1972) applies verbatim. Thus, by Corollary 2 above, together with what was noted in the proof of Proposition 3 about the indefinite Bochner integral, blocking power of small coalitions as in the result of Schmeidler (1972) can be deduced if the commodity space is any infinite-dimensional Banach space, provided that allocations are Bochner integrable and that the space of agents is modeled as an $\omega_{1}$-atomless totally finite measure space. ${ }^{7}$

There are contexts in which blocking power of small coalitions can also be demonstrated using our results when allocations are Gelfand integrable functions with values in a dual Banach space. An important case where allocations are taken to be Gelfand integrable arises in treatments of commodity differentiation where consumption vectors are described by the non-negative elements of $M(K)$, the space of bounded signed Borel measures on a compact metric space $K$, which is the dual of the space $C(K)$ of continuous functions on $K$, with the sup-norm. Write $M(K)_{+}$for the cone of nonnegative elements of $M(K)$. Let $(T, \Sigma, \mu)$ be a totally finite measure space of agents. Note that if $f$ is any Gelfand integrable function from $T$ to $M(K)_{+}$, then, writing $\|\cdot\|$ for the variation norm of $M(K)$, the function $t \mapsto\|f(t)\|$ is integrable, because $\|f(t)\|=\int_{K} 1_{K} \mathrm{~d} f(t)$. Thus, by Lemma 4, a Gelfand integrable function from $T$ to $M(K)_{+}$defines a $\mu$-continuous (norm-) countably additive vector measure on $\Sigma$. Now, $K$ being a compact metric space, $C(K)$ is separable. Thus, by Corollary 5 above, a result as in Schmeidler (1972) on blocking power of small coalitions can also be deduced in the just sketched framework of commodity differentiation if the measure $\mu$ on the set of agents is $\omega_{1}$-atomless. ${ }^{8}$

It should be mentioned that for $\ell_{\infty}$ as commodity spaces, Hervés-Beloso et al. (1996) have established an extension of Schmeidler's result under the usual hypothesis of non-atomicity of the space of agents, without using a version of Liapounoff's theorem for infinite-dimensional spaces. What drives this result in Hervés-Beloso et al. (1996) is, in particular, the assumption that preferences be Mackey continuous, which

\footnotetext{
7 A similar observation was also made in Khan and Sagara (2013 Theorem 6.2).

8 This is not possible by appealing to Khan and Sagara (2013); see Remark 3 above.
} 
makes it possible to use an approximating sequence of finite-dimensional subspaces. In contrast, the argument in Schmeidler does not rely on any continuity hypothesis on preferences.

\subsection{Core-Walras equivalence in coalitional exchange economies}

Let $(T, \Sigma, \mu)$ be a totally finite measure space of agents, and let the commodity space be an order-continuous Banach lattice $X$ with positive cone $X_{+}$.

A coalitional exchange economy for $(T, \Sigma, \mu)$ and $X$ is described as follows. Allocations are $\mu$-continuous countably additive vector measures $G: \Sigma \rightarrow X_{+}$. We write $\mathcal{A}$ for the space of all such allocations, and $\Omega$ for the initial allocation. Members of $\Sigma$ are called coalitions. For each coalition $A$ there is a preference relation $\succ_{A}$ on $\mathcal{A}$. An allocation $G$ is feasible if $G(T)=\Omega(T)$. A coalition $A$ can block an allocation $G$ if there is an allocation $F$ such that $F \succ_{A} G$ and $F(A)=\Omega(A)$. An allocation is a core allocation if it is feasible and cannot be blocked by any coalition $A$ with $\mu(A)>0$. The allocation $G$ is called Walrasian if it is feasible and if there is a nonzero $p^{*} \in X^{*}$ such that for each coalition $A$ with $\mu(A)>0, p^{*} G(A) \leq p^{*} \Omega(A)$ and $p^{*} F(A)>p^{*} \Omega(A)$ whenever $F$ is an allocation with $F \succ_{A} G$.

We summarize the data defining a coalitional exchange economy as just described by a list $\mathcal{E}=\left((T, \Sigma, \mu),\left\langle\succ_{A}\right\rangle_{A \in \Sigma}, \Omega\right)$. In the sequel, for $x, y \in X, x>y$ means $x \geq y$ and $x \neq y$.

The following assumptions are standard and natural when economies are described in coalitional form.

(P1) For every $A \in \Sigma, \succ_{A}$ is irreflexive and transitive.

(P2) For every $A \in \Sigma$, if $F \succ_{A} G$ then $F \succ_{B} G$ for every $B \in \Sigma$ with $B \subseteq A$ and $\mu(B)>0$.

(P3) For every $A \in \Sigma$ and any $F, F^{\prime}, G, G^{\prime} \in \mathcal{A}$, if $F \succ_{A} G$ and if for all $B \in \Sigma$, $F(B \cap A)=F^{\prime}(B \cap A)$ and $G(B \cap A)=G^{\prime}(B \cap A)$, then $F^{\prime} \succ_{A} G^{\prime}$.

(P4) For every $A, B \in \Sigma$, if $F \succ_{A} G$ and $F \succ_{B} G$ then $F \succ_{A \cup B} G$.

(P5) For every $A \in \Sigma$ with $\mu(A)>0$, and any $F, G \in \mathcal{A}$, if $F(B)>G(B)$ for every $B \in \Sigma$ with $B \subseteq A$ and $\mu(B)>0$, then $F \succ_{A} G$.

The next assumption is taken from Zame (1986).

(P6) There are strictly positive elements $\alpha^{*}, \beta^{*} \in X^{*}$, with $\beta^{*} \geq \alpha^{*}$, such that for every $A \in \Sigma$ with $\mu(A)>0$, and any $F, G, H \in \mathcal{A}$, if $F(B) \geq G(B)$ for all $B \in \Sigma$ and $\alpha^{*} H(B)>\beta^{*} G(B)$ for all $B \in \Sigma$ with $B \subseteq A$ and $\mu(B)>0$, then $F-G+H \succ_{A} F$.

This assumption puts bounds on marginal rates of substitution. It is well known that assumptions on marginal rates of substitution are needed in contexts of infinitedimensional commodity spaces when consumption sets may have empty interior.

Our final assumption is implied by condition (C-6) in Zame (1986). It imposes some kind of lower semi-continuity property on preferences. In our proof, we use it to show that a quasi-equilibrium is a Walrasian equilibrium. 
(P7) For every $A \in \Sigma$ with $\mu(A)>0$, any $F, G \in \mathcal{A}$ with $F \succ_{A} G$, and any $\epsilon>0$, there exist $\gamma \in(0,1)$ and $A^{\prime} \in \Sigma$ with $A^{\prime} \subseteq A$ such that $\mu\left(A^{\prime}\right)>\mu(A)-\epsilon$ and $\gamma F \succ_{A^{\prime}} G$.

Remark 12 The way we define coalitional exchange economies follows Zame (1986) and differs slightly from Vind (1964), where allocations need not be $\mu$-continuous with respect to some population measure $\mu$. There seems to be no economic reason for rejecting the existence of such a population measure though.

The core-Walras equivalence result for coalitional economies in Zame (1986) makes assumptions in addition to what is contained in (P1)-(P7). In particular, it is assumed in that result that the range of the initial allocation is a relatively norm-compact subset of $X_{+}$. As pointed out in Zame (1986), if this assumption is not satisfied, then the set of core allocations of an atomless coalitional economy may be strictly larger than the set of Walrasian allocations. Our proposition below shows that if the non-atomicity hypothesis on the measure space of agents is strengthened, so that our version of Liapounoff's theorem applies, then core-Walras equivalence holds without any assumption on the range of the initial allocation (see also Remark 13 below).

Proposition 4 Let $\mathcal{E}=\left((T, \Sigma, \mu),\left\langle\succ_{A}\right\rangle_{A \in \Sigma}, \Omega\right)$ be a coalitional exchange economy with commodity space $X$, where $X$ is an order-continuous Banach lattice. Suppose $\mathcal{E}$ satisfies $(\mathrm{P} 1)-(\mathrm{P} 7)$ and that for some uncountable cardinal $\kappa, \mu$ is $\kappa$-atomless with $\kappa>\operatorname{dens}(X)$. Then the set of core allocations of $\mathcal{E}$ coincides with the set of Walrasian allocations of $\mathcal{E}$.

For convenience, two steps of the proof of this proposition are singled out as lemmas.

Lemma 5 Let $(T, \Sigma, \mu)$ be a totally finite measure space, $X$ a Banach space, and $F_{1}, F_{2}: \Sigma \rightarrow X$ two $\mu$-continuous countably additive vector measures. Let $A_{1}, A_{2} \in$ $\Sigma$ and $0<\alpha<1$. Suppose that any of the conditions (a)-(c) of Lemma 3 holds. Then there are elements $B_{1}, B_{2} \in \Sigma$, with $B_{1} \subseteq A_{1}, B_{2} \subseteq A_{2}$, and $B_{1} \cap B_{2}=\emptyset$, such that $F_{1}\left(B_{1}\right)+F_{2}\left(B_{2}\right)=\alpha F_{1}\left(A_{1}\right)+(1-\alpha) F_{2}\left(A_{2}\right)$.

Proof From Corollaries 3, 1, and 4, respectively, it follows that there are elements $C_{1}, C_{2} \in \Sigma$, with $C_{1} \subseteq A_{1} \backslash A_{2}$ and $C_{2} \subseteq A_{2} \backslash A_{1}$, such that $F_{1}\left(C_{1}\right)=\alpha F_{1}\left(A_{1} \backslash A_{2}\right)$ and $F_{2}\left(C_{2}\right)=(1-\alpha) F_{2}\left(A_{2} \backslash A_{1}\right)$. By Lemma 3 there is a $C_{3} \subseteq A_{1} \cap A_{2}$ such that $F_{1}\left(C_{3}\right)+F_{2}\left(\left(A_{1} \cap A_{2}\right) \backslash C_{3}\right)=\alpha F_{1}\left(A_{1} \cap A_{2}\right)+(1-\alpha) F_{2}\left(A_{1} \cap A_{2}\right)$. Take $B_{1}=C_{1} \cup C_{3}$ and $B_{2}=C_{2} \cup\left(\left(A_{1} \cap A_{2}\right) \backslash C_{3}\right)$.

Lemma 6 Let $(T, \Sigma, \mu)$ be a totally finite measure space, $X$ an order-continuous Banach lattice, and $F: \Sigma \rightarrow X_{+}$a $\mu$-continuous countably additive vector measure. Then given $A \in \Sigma$ and given $b \in X$ with $0 \leq b \leq F(A)$, there is a $\mu$-continuous countably additive vector measure $G: \Sigma \rightarrow X_{+}$such that $G(A)=b$ and $G(B) \leq$ $F(B)$ for all $B \in \Sigma$.

Proof Let $\mathcal{P}$ be the set of all finite subalgebras of $\Sigma$ which contain $A$ as an element. Write $a$ for generic members of $\mathcal{P}$. Each $a$ is generated by a finite partition of $T$ into elements of $\Sigma$. Therefore, using the Riesz decomposition theorem, for each $a \in \mathcal{P}$ 
we can find an additive function $g_{a}: a \rightarrow X_{+}$such that $g_{a}(A)=b$ and such that $g_{a}(B) \leq F(B)$ for all $B \in \Sigma$. Extend each $g_{a}$ to a function $f_{a}: \Sigma \rightarrow X_{+}$by setting $f_{a}(B)=0$ for $B \in \Sigma$ with $B \notin a$. Then for each $a \in \mathcal{P}$ and each $B \in \Sigma, f_{a}(B)$ belongs to the order interval $[0, F(B)]$. Note that $(\mathcal{P}, \subseteq)$ is upwards directed. Thus the family $\left\langle f_{a}\right\rangle_{a \in \mathcal{P}}$ is a net in the product $\prod_{B \in \Sigma}[0, F(B)]$ of order intervals of $X$. As the Banach lattice $X$ is order-continuous, order intervals in $X$ are weakly compact, so by Tychonoff's theorem we may assume, passing to a subnet of $\left\langle f_{a}\right\rangle_{a \in \mathcal{P}}$ if necessary, that there is a $G: \Sigma \rightarrow X_{+}$, with $G(B) \leq F(B)$ for all $B \in \Sigma$, such that $f_{a}(B) \rightarrow G(B)$ for all $B \in \Sigma$ weakly in $X$. In particular, we must have $G(A)=b$. Pick any $B, C \in \Sigma$ with $B \cap C=\emptyset$. There is an $a \in \mathcal{P}$ such that both $B$ and $C$ are in $a^{\prime}$ for all $a^{\prime} \in \mathcal{P}$ with $a \subseteq a^{\prime}$. By construction, $f_{a^{\prime}}(B)+f_{a^{\prime}}(C)=f_{a^{\prime}}(B \cup C)$ for such $a^{\prime}$. Hence, in the limit, $G(B)+G(C)=G(B \cup C)$ (as the weak topology of $X$ is a linear space topology). Thus $G$ is additive. Now, $X$ being a Banach lattice, $0 \leq G(B) \leq F(B)$ implies $\|G(B)\| \leq\|F(B)\|$ for all $B \in \Sigma$. Consequently, $\mu$-continuity of $F$ implies that $G$ is $\mu$-continuous as well. In particular, being additive, $G$ must actually be countably additive.

Proof of Theorem 4 Clearly every Walrasian allocation belongs to the core. For the reverse implication, suppose $\tilde{F}$ is a core allocation and let

$$
\mathcal{Z}=\left\{F(A)-\Omega(A): F \in \mathcal{A}, A \in \Sigma, F \succ_{A} \tilde{F}\right\} \cup\{0\} .
$$

Then $\mathcal{Z}$ is non-empty. We claim that $\mathcal{Z}$ is convex. To see this, note first that $\mu$-continuity of allocations, Corollary 1, and (P2) together imply $\alpha(F-\Omega)(A) \in \mathcal{Z}$ for $0<\alpha<1$ whenever $F \succ_{A} \tilde{F}$ for $F \in \mathcal{A}$ and $A \in \Sigma$. Now let $F_{1}, F_{2} \in \mathcal{A}$, let $A_{1}, A_{2} \in \Sigma$, and suppose that $F_{1} \succ_{A_{1}} \tilde{F}$ and $F_{2} \succ_{A_{2}} \tilde{F}$. Let $0<\alpha<1$. By Lemma 5 there are $B_{1}, B_{2} \in \Sigma$, with $B_{1} \subseteq A_{1}, B_{2} \subseteq A_{2}$, and $B_{1} \cap B_{2}=\emptyset$, such that

$$
\left(F_{1}-\Omega\right)\left(B_{1}\right)+\left(F_{2}-\Omega\right)\left(B_{2}\right)=\alpha\left(F_{1}-\Omega\right)\left(A_{1}\right)+(1-\alpha)\left(F_{2}-\Omega\right)\left(A_{2}\right) .
$$

We may assume that both $\mu\left(B_{1}\right)>0$ and $\mu\left(B_{2}\right)>0$; otherwise, because allocations are $\mu$-continuous, the situation reduces to a similar one as above. Define $F_{3} \in \mathcal{A}$ by setting $F_{3}(C)=F_{1}\left(C \cap B_{1}\right)+F_{2}\left(C \cap B_{2}\right)$ for every $C \in \Sigma$. Now (P2)-(P4) together imply $F_{3} \succ_{B_{1} \cup B_{2}} \tilde{F}$, and thus we have $\left(F_{3}-\Omega\right)\left(B_{1} \cup B_{2}\right) \in \mathcal{Z}$. Moreover, $\left(F_{3}-\Omega\right)\left(B_{1} \cup B_{2}\right)=\left(F_{1}-\Omega\right)\left(B_{1}\right)+\left(F_{2}-\Omega\right)\left(B_{2}\right)$, and we conclude that $\mathcal{Z}$ is convex.

Now let $\Lambda=\left\{x \in X: \alpha^{*}\left(x^{-}\right)>\beta^{*}\left(x^{+}\right)\right\}$, where $\alpha^{*}$ and $\beta^{*}$ are the elements of $X^{*}$ from (P6). We claim that $\mathcal{Z} \cap \Lambda=\emptyset$. To see this, suppose by way of contradiction that there are an $F \in \mathcal{A}$ and an $A \in \Sigma$ such that $F \succ_{A} \tilde{F}$ and such that, writing $a=(F(A)-\Omega(A))^{-}$and $b=(F(A)-\Omega(A))^{+}$, we have $\alpha^{*}(a)>\beta^{*}(b)$. Note that this implies $\mu(A)>0$ (as allocations are $\mu$-continuous).

Suppose $b=0$ and note that this means $a=-(F(A)-\Omega(A))$. Define $G \in \mathcal{A}$ by setting $G(B)=\frac{\mu(B)}{\mu(A)} a$ for each $B \in \Sigma$. Then $(F+G)(A)=\Omega(A)$, and by $(\mathrm{P} 1)$ and (P5), $F+G \succ_{A} \tilde{F}$, contradicting the hypothesis that $\tilde{F}$ is a core allocation.

Thus suppose $b \neq 0$ and note that this means $\beta^{*}(b)>0$ as $\beta^{*}$ is strictly positive. Appealing to Lemma 6 , choose a $G \in \mathcal{A}$ such that $G(A)=b$ and such that $F(B) \geq$ 
$G(B)$ for all $B \in \Sigma$. Choose $0<\gamma<1$ such that $\alpha^{*}(\gamma a)>\beta^{*}(b)$ and define $H \in \mathcal{A}$ by setting $H(B)=\frac{\beta^{*}(G(B))}{\beta^{*}(b)} \gamma a+\frac{\mu(B)}{\mu(A)}(1-\gamma) a$ for all $B \in \Sigma$, so that $\alpha^{*} H(B)>\beta^{*} G(B)$ if $\mu(B)>0$. By (P6) and (P1), $F-G+H \succ_{A} \tilde{F}$. On the other hand, as $G(A)=b$, we have $H(A)=a$, and since $b-a=F(A)-\Omega(A)$ by the definition of $a$ and $b$, it follows that $(F-G+H)(A)=F(A)-b+a=\Omega(A)$, and we get again a contradiction to the hypothesis that $\tilde{F}$ belongs to the core.

Finally, $\Lambda$ is non-empty, open, and convex, and $\Lambda \cup\{0\}$ is a cone. For the first of these properties, note that $-X_{+} \backslash\{0\} \subseteq \Lambda$. For the others, note that $\Lambda=\rho^{-1}((-\infty, 0))$ where $\rho: X \rightarrow \mathbb{R}$ is given by $\rho(x)=\left(\beta^{*}-\alpha^{*}\right)\left(x^{+}\right)+\alpha^{*}(x)$. Clearly the function $\rho$ is positively homogeneous. By continuity of the lattice operations, $\rho$ is continuous, and as $\beta^{*}-\alpha^{*} \geq 0$ and $(x+y)^{+} \leq x^{+}+y^{+}$for any $x, y \in X, \rho$ is subadditive.

In view of the facts noted so far, we may appeal to the separation theorem to find a $p^{*} \in X^{*}$ with $p^{*} \neq 0$ such that $p^{*} x \leq p^{*} z$ for each $x \in \Lambda$ and $z \in \mathcal{Z}$. As noted above, $-X_{+} \backslash\{0\} \subseteq \Lambda$. Consequently, as $\Lambda$ is open and $\Lambda \cup\{0\}$ is a cone, $p^{*}$ must be strictly positive. Also by the fact that $\Lambda \cup\{0\}$ is a cone, we must have $p^{*} z \geq 0$ for each $z \in \mathcal{Z}$. By the definition of $\mathcal{Z}$, this means that for every $A \in \Sigma$, if $F \in \mathcal{A}$ and $F \succ_{A} \tilde{F}$ then $p^{*} F(A) \geq p^{*} \Omega(A)$.

In particular, we have $p^{*} \tilde{F}(A)=p^{*} \Omega(A)$ for all $A \in \Sigma$. For if not, feasibility and additivity of $\tilde{F}$ imply existence of an $A \in \Sigma$ with $p^{*} \tilde{F}(A)<p^{*} \Omega(A)$. For such an $A, \mu(A)>0$, and for some $x \in X_{+} \backslash\{0\}, p^{*}(\tilde{F}(A)+x)<p^{*} \Omega(A)$. Define $F \in \mathcal{A}$ by setting $F(B)=\tilde{F}(B)+\frac{\mu(B)}{\mu(A)} x$ for all $B \in \Sigma$. By (P5), $F \succ_{A} \tilde{F}$, and we get a contradiction to the conclusion of the previous paragraph.

Fix any $A \in \Sigma$ with $\mu(A)>0$. To finish the proof, we need to show that we actually have $p^{*} F(A)>p^{*} \Omega(A)$ for any $F \in \mathcal{A}$ with $F \succ_{A} \tilde{F}$. If $p^{*} \Omega(A)=0$ this holds because $\succ_{A}$ is irreflexive and $p^{*}$ is strictly positive. Suppose $p^{*} \Omega(A)>0$ and that for some $F \in \mathcal{A}, F \succ_{A} \tilde{F}$ but $p^{*} F(A)=p^{*} \Omega(A)$. Combining (P7) and the fact that $B \mapsto p^{*} \Omega(B)$ is $\mu$-continuous on $\Sigma$, we may find a $0<\gamma<1$ and an $A^{\prime} \in \Sigma$ with $A^{\prime} \subseteq A$ such that both $\gamma F \succ_{A^{\prime}} \tilde{F}$ and $p^{*} \Omega\left(A^{\prime}\right)>0$. By (P2), we also have $F \succ_{A^{\prime}} \tilde{F}$, and in addition, $F \succ_{A \backslash A^{\prime}} \tilde{F}$ if $\mu\left(A \backslash A^{\prime}\right)>0$. Thus, by additivity of $F$ and $\Omega$, the hypothesis that $p^{*} F(A)=p^{*} \Omega(A)$ and the last sentence of the penultimate paragraph together imply that $p^{*} F\left(A^{\prime}\right)=p^{*} \Omega\left(A^{\prime}\right)$. As $p^{*} \Omega\left(A^{\prime}\right)>0$, it follows that $p^{*} \gamma F\left(A^{\prime}\right)<p^{*} \Omega\left(A^{\prime}\right)$. But this together with $\gamma F \succ_{A^{\prime}} \tilde{F}$ contradicts the last sentence of the penultimate paragraph, and this contradiction completes the proof.

Remark 13 Let $(T, \Sigma, \mu)$ be a totally finite measure space of agents, and take an infinite-dimensional Banach space $X$ as commodity space. Net-trades can be approximated by commodity vectors belonging to some dense subset of $X$, and coalitions can be approximated by coalitions belonging to some subset of $\Sigma$ which is dense for the pseudo-metric on $\Sigma$ coming from the norm of $L_{1}(\mu)$. Thus, by Fact 1 stated in Sect. 2, the condition in Proposition 4 that for some uncountable cardinal $\kappa, \mu$ is $\kappa$-atomless with $\kappa>\operatorname{dens}(X)$ may be interpreted as requiring that there be "many more coalitions than commodities."

The original assumption that the range of the initial allocation of an economy be norm-compact was identified by Zame (1986) with "thick" markets. Thus Proposition 4 
shows that core-Walras equivalence in a coalitional economy can be deduced without "thick markets" if the "number" of coalitions in the economy is "large enough."

Remark 14 For the individualistic setting of atomless economies as introduced by Aumann (1964), Tourky and Yannelis (2001) have identified a condition requiring "many more agents than commodities" as crucial for core-Walras equivalence. Mathematically, this condition may be formalized-with $(T, \Sigma, \mu)$ and $X$ as in the previous remark - as requiring add $\mathcal{N}(\mu)>\operatorname{dens}(X)$, where add $\mathcal{N}(\mu)$ is the additivity of the ideal $\mathcal{N}(\mu)$ of $\mu$-null sets, i.e., the least cardinal of any family of $\mu$-null sets whose union is not a $\mu$-null set. The conditions of "many more agents than commodities" and of "many more coalitions than commodities" are not comparable. E.g., if $\mu$ is Lebesgue measure on $[0,1]$, then add $\mathcal{N}(\mu) \geq \omega_{1}$, but $\mu$ is not $\omega_{1}$-atomless, so that if $X$ is separable, the former condition holds, but the latter fails. On the other hand, if $\mu$ is the usual measure on $\{0,1\}^{\omega_{2}}$, then $\mu$ is $\omega_{2}$-atomless, but add $\mathcal{N}(\mu)=\omega_{1}$ (see Fremlin $2008523 \mathrm{E}$ ), so that if $\operatorname{dens}(X)=\omega_{1}$, the latter condition holds, but the former fails. The non-equivalence of the two conditions is just another manifestation of the fact, discussed in Gretsky and Ostroy (1985) and Zame (1986), that with infinitedimensional commodity spaces the individualistic and the coalitional representation of large economies are in general not equivalent.

\section{References}

Aumann, R.J.: Markets with a continuum of traders. Econometrica 32, 39-50 (1964)

Diestel, J., Uhl, J.J., Jr.: Vector Measures. American Mathematical Society, Providence (1977) (with a foreword by B. J. Pettis, Mathematical Surveys, No. 15)

Easton, W.B.: Powers of regular cardinals. Ann Math Logic 1, 139-178 (1970)

Fabian, M., Habala, P., Hájek, P., Montesinos Santalucía, V., Pelant, J., Zizler, V.: Functional analysis and infinite-dimensional geometry. In: CMS Books in Mathematics/Ouvrages de Mathématiques de la SMC, vol. 8. Springer, Berlin (2001)

Fremlin, D.H.: Measure Theory, vol. 3. Measure algebras. Torres Fremlin, Colchester (2004) (corrected second printing of the 2002 original)

Fremlin, D.H.: Measure theory, vol. 5. In: Set-Theoretic Measure Theory. Part I. Torres Fremlin, Colchester (2008)

Gretsky NE, Ostroy JM (1985) Thick and thin market nonatomic exchange economies. In: Advances in Equilibrium Theory (Indianapolis, Ind., 1984). Lecture Notes in Economics and Mathematical Systems, vol. 244, pp. 107-129. Springer, Berlin

Hervés-Beloso, C., Moreno-García, E., Núñez-Sanz, C., Páscoa, M.R.: Blocking efficacy of small coalitions in myopic economies. J. Econ. Theory 93(1), 72-86 (2000). doi:10.1006/jeth.2000.2647. http://www. sciencedirect.com/science/article/pii/S00220531009

Hoover, D.N., Keisler, H.J.: Adapted probability distributions. Trans. Am. Math. Soc. 286(1), 159-201 (1984). doi:10.2307/1999401

Keisler, H.J., Sun, Y.: Why saturated probability spaces are necessary. Adv. Math. 221(5), 1584-1607 (2009). doi:10.1016/j.aim.2009.03.003

Khan, M., Sagara, N.: Maharam-types and Lyapunov's theorem for vector measures on Banach spaces. Illinois J. Math. (2013) (in press)

Kluvánek, I.: The range of a vector-valued measure. Math. Syst. Theory 7, 44-54 (1973)

Knowles, G.: Lyapunov vector measures. SIAM J. Control 13, 294-303 (1975)

Lindenstrauss, J.: A short proof of Liapounoff's convexity theorem. J. Math. Mech. 15, 971-972 (1966)

Mackey, G.W.: On infinite-dimensional linear spaces. Trans. Am. Math. Soc. 57, 155-207 (1945)

Maharam, D.: On homogeneous measure algebras. Proc. Natl. Acad. Sci. USA 28, 108-111 (1942)

Podczeck, K.: On the convexity and compactness of the integral of a Banach space valued correspondence. J. Math. Econom. 44(7-8), 836-852 (2008). doi:10.1016/j.jmateco.2007.03.003 
Richter, H.: Verallgemeinerung eines in der Statistik benötigten Satzes der Masstheorie. Math. Ann. 150, 85-90 (1963)

Rosenthal, H.P.: On injective Banach spaces and the spaces $C(S)$. Bull. Am. Math. Soc. 75, 824-828 (1969)

Rustichini, A., Yannelis, N.C.: What is perfect competition? In: Yannelis, N.C., Khan, M.A. (eds.) Equilibrium Theory in Infinite Dimensional Spaces. Springer, Berlin (1991)

Schmeidler, D.: A remark on the core of an atomless economy. Econometrica 40, 579-580 (1972)

Scott, D.: A proof of the independence of the continuum hypothesis. Math. Syst. Theory 1, 89-111 (1967)

Tourky, R., Yannelis, N.C.: Markets with many more agents than commodities: Aumann's "hidden" assumption. J. Econ. Theory 101, 189-221 (2001)

Uhl, J.J., Jr.: The range of a vector-valued measure. Proc. Am. Math. Soc. 23, 158-163 (1969)

Vind, K.: Edgeworth-allocations in an exchange economy with many traders. Int. Econ. Rev. 5(2), 165-177 (1964)

Yannelis, N.C.: Integration of Banach-valued correspondences. In: Khan, M.A., Yannelis, N.C. (eds.) Equilibrium Theory in Infinite Dimensional Spaces, Studies in Economic Theory, vol. 1, pp. 2-35. Springer, Berlin (1991). doi:10.1007/978-3-662-07071-01

Zame, W.R.: Markets with a continuum of traders and infinitely many commodities. Working paper, SUNY at Buffalo (1986) 